\title{
CENÁRIO DA ENFERMAGEM FORENSE NA FORMAÇÃO DO ENFERMEIRO NA ASSISTÊNCIA E NA PESQUISA
}

Jhuliano Silva Ramos de Souza ${ }^{1}$ Andreia Cristina Barbosa Costa ${ }^{1}$ Sueli de Carvalho Vilela ${ }^{1}$ https://orcid.org/0000-0002-4338-4433

https://orcid.org/0000-0003-3484-9638

http://orcid.org/0000-0003-3034-3904

Objetivo: analisar como o cenário da enfermagem forense contribui para a formação do enfermeiro na assistência e na pesquisa. Método: trata-se de uma revisão integrativa, sendo utilizadas 6 bases de dados, com descritores forensic nursing, education, nursing, currículum, enfermagem forense, educação em enfermagem, currículo, totalizando uma amostra final de 13 artigos. Resultados: o nível de evidências dos estudos baseou-se em opiniões de especialistas e em pesquisas qualitativas, nos quais surgiram duas categorias relacionadas ao ensino da enfermagem forense, modificando o cenário científico e assistencial da enfermagem; e o cenário da enfermagem forense como especialidade nova na enfermagem. Conclusão: quanto à formação, observa-se que, embora seja uma especialidade nova, ainda permanece como uma área pouco explorada pelos enfermeiros. Com isso, acredita-se que é pertinente aprofundar o estudo sobre como a enfermagem forense tem se consolidado no ensino, bem como as possiveis lacunas existentes nesse setor.

Descritores: Enfermagem Forense; Educação em Enfermagem; Currículo.

\section{SCENARIO OF FORENSIC NURSING IN NURSING TRAINING IN ASSISTANCE AND RESEARCH}

Objective: analyze how the scenario of forensic nursing contributes to the training of nurses in care and research. Method: it is an integrative review, using 6 databases, with descriptors forensic nursing, education, nursing, curriculum, forensic nursing, nursing education, curriculum, forensic nursing, nursing education and curriculum, totaling for the final sample of 13 articles. Results: the level of evidence in the studies was based on expert opinions and qualitative research, in which two categories related to the teaching of forensic nursing emerged, changing the scientific and care scenario of nursing; and the scenario of forensic nursing as a new specialty in nursing. Conclusion: regarding training, it is observed that, although it is a new specialty, it still remains an area little explored by nurses. With this, it is believed that it is pertinent to deepen the study on how forensic nursing has been consolidated in teaching, as well as the possible gaps in this sector Descriptors: Forensic Nursing; Education, Nursing; Curriculum.

\section{ESCENARIO DE ENFERMERİA FORENSE EN FORMACIÓN EN ENFERMERÍA EN ASISTENCIA E INVESTIGACIÓN}

Objetivo: analizar cómo el escenario de la enfermería forense contribuye a la formación de enfermeras en atención e investigación. Método: es una revisión integradora, que utiliza 6 bases de datos, con descriptores de enfermería forense, educación, enfermería, currículo, enfermería forense, educación en enfermería, currículo, enfermería forense, educación en enfermería y currículum, totalizando para la muestra final de 13 artículos. Resultados: el nivel de evidencia en los estudios se basó en opiniones de expertos e investigaciones cualitativas, en las que surgieron dos categorías relacionadas con la enseñanza de la enfermería forense, que cambiaron el escenario científico y asistencial de la enfermería; y el escenario de la enfermería forense como una nueva especialidad en enfermería. Conclusión: con respecto a la capacitación, se observa que, aunque es una nueva especialidad, sigue siendo un área poco explorada por las enfermeras. Con esto, se cree que es pertinente profundizar el estudio sobre cómo la enfermería forense se ha consolidado en la enseñanza, así como las posibles brechas en este sector.

Descriptores: Enfermería forense; Educación en Enfermería; Curriculum.

${ }^{1}$ Universidade Federal de Alfenas, MG.

Autor Correspondente: Jhuliano Silva Ramos de Souza Email: jhulianoramoz@hotmail.com

Recebido:18/2/2020 Aceito:21/7/2020 


\section{INTRODUÇÃO}

A enfermagem contemporânea tenta romper com o modelo tecnicista e mecanicista, abraçando um paradigma envolto nos aspectos sociais, humanísticos, éticos e interpessoais como processos fundamentais de quem necessita de cuidados. Isso não poderia ser diferente na formação dos enfermeiros, carecendo de inserções e de mudanças no âmbito acadêmico profissional ${ }^{1}$.

A enfermagem forense (EF) mostra-se em destaque como uma nova especialidade reconhecida legalmente em 1995 pela Associação Americana de Enfermeiros $(A N A)^{2}$ e, no Brasil, pelo Conselho Federal de Enfermagem (COFEN) na resolução no 389, de 18 de outubro de $2011^{3}$. Essa especialidade tem como vertente a violência. Assim, os enfermeiros lidam com pacientes vítimas de violência e os perpetradores, utilizando seus saberes científicos de enfermagem com o alinhamento das ciências forenses ${ }^{4}$. Nessa ótica, abrange o papel essencial no cuidado social, psicológico e humanístico do indivíduo vulnerável à violência e, não exclusivamente, os procedimentos clínicos/tecnicistas, científicos e legais ${ }^{5}$

Nesse sentido, o Brasil torna-se um campo fecundo para a EF, uma vez que destaca-se como o país em que há grande incidência de violência nas diversas categorias e evidencia as causas de morte, como os homicídios por arma de fogo, violência contra negros, violência contra a mulher, dentre outros ${ }^{6}$.

No contexto educacional ${ }^{7}$, demonstra sua importância em programas de ensino de enfermagem e na educação continuada tanto em cursos de graduação como de pós-graduação em enfermagem. Os efeitos positivos da $\mathrm{EF}^{7}$ estão relacionados a diminuição da sobrecarga no sistema de saúde, os benefícios no atendimento e na segurança das pessoas, tanto no cuidado ao indivíduo e aos familiares como ao profissional ${ }^{1}$.

Diante do exposto, este trabalho tem por objetivo analisar como o cenário da enfermagem forense contribui para a formação do enfermeiro na assistência e na pesquis

\section{MÉTODO}

\section{Tipo de estudo}

Trata-se de uma revisão integrativa da literatura em que foram percorridas as seguintes etapas: problema e objetivo de pesquisa; busca na literatura; critérios de inclusão e de exclusão; análise dos artigos; apresentação e discussão dos resultados ${ }^{8}$. Iniciou-se com a definição do problema por meio da questão norteadora: Quais as contribuições do cenário da enfermagem forense para a formação do enfermeiro na assistência e na pesquisa?

\section{Critérios de inclusão e exclusão}

Empregaram-se como critérios de inclusão artigos na íntegra, disponiveis online e um recorte temporal dos últimos 10 anos (2008 a 2018). Optou-se por utilizar esse recorte em vista em analisar as publicações atualizadas sobre a temática, uma vez que a formação do enfermeiro tanto na assistência quanto na pesquisa é dinâmica. Foram excluídos artigos duplicados, dissertações de mestrado, teses de doutorado, estudo de casos clínicos e artigos que trouxessem o papel de outros profissionais na prática forense.

\section{Procedimento de coleta de dados e análise dos da-} dos

A coleta foi realizada em agosto de 2018 nas seguintes bases de dados/banco eletrônicos, através do portal da Coordenação de Aperfeiçoamento de Pessoal de Nivel Superior (CAPES) e da Biblioteca Virtual em Saúde (BVS): Web Of Science (WOS); ScOPUS (Elsevier); Cummulative Index to Nursing and Allied Health Literature (CINAHL); Cientific Electronic Library Online (SCIELO) e Medical Literature and Retrivial Sistem on Line (MEDLINE) e Literatura Latino Americana e do Caribe em Ciências da Saúde (LILACS).

Para tal, utilizaram-se os cruzamentos dos Descritores Ciência da Saúde (DECS) e Medical Subject Headings da U.S. National Library of Medicine (Mesh): Forensic Nursing AND Education, nursing; Forensic Nursing AND Currículum; Forensic Nursing AND Currículum AND Education, nursing: Enfermagem Forense AND Educação em Enfermagem; Enfermagem Forense AND Currículo; Enfermagem Forense AND Educação em Enfermagem AND Currículo.

O nível de evidência baseou-se na classificação de seis níveis, sendo o primeiro relacionado a revisões sistemáticas e de metanálise; o segundo, quanto a estudos experimentais e de pesquisas clínicas; o terceiro, a estudos quase experimentais; o quarto, referente a estudos descritivos ou qualitativos; o quinto, a relato de experiências; por fim, o sexto baseou-se em opiniões de especialistas?

Criou-se um banco de dados, o qual foram contemplados os critérios: título; autor; ano; publicação; fonte; objeto; metodologia; resultado; conclusão e nível de evidência. O conteúdo foi interpretado por meio de categorização na vertente de Bardim ${ }^{10}$. 


\section{RESULTADOS}

A busca bibliográfica resultou em 982 estudos na primeira seleção feita pelas leituras de títulos e de resumos. Da busca, excluíram-se os artigos duplicados (140) e aqueles que não atenderam à pergunta norteadora (809) por serem trabalhos sobre estudos voltados a ensaios clínicos, temas relacionados à enfermagem forense no atendimento clínico e estudos relacionados às ciências forenses que não se enquadravam com a temática.
A partir da primeira análise, 33 estudos foram pré-selecionados, dentre os quais foram lidos na integra. Desses, 16 concentrados na MEDLINE; 3 na CINAHL; e I na WOS, não atenderam o objetivo do estudo, por se tratarem de temas referentes a abuso de idosos, simulação realística na prática, ética em enfermagem, mercado de trabalho na enfermagem, evolução histórica da enfermagem, sendo excluídos após análise criteriosa. Assim, a amostra resultou em 13 artigos, como evidencia o diagrama Prismall abaixo:

Figura 1 - Processo de seleção dos estudos encontrados pelas bases de dados, elaborado de acordo com o Diagrama Prisma.
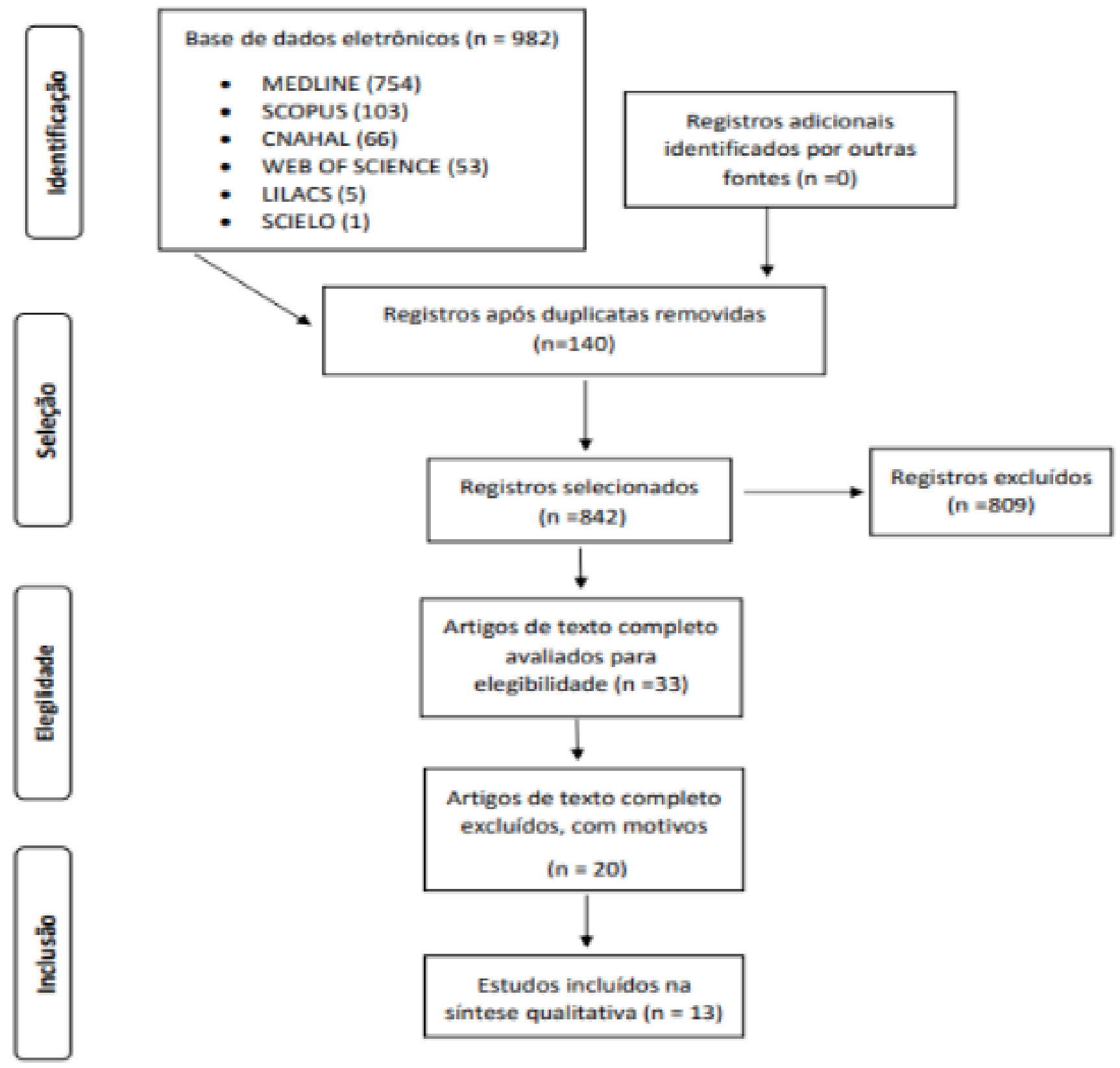

Fonte: elaborado pelos autores conforme o Diagrama de PRISMA. 
Quadro 1 - Distribuição dos artigos selecionados, segundo autor/ano, título, periódico, objetivo, método, resultados e nível de evidência. Alfenas-MG, 2019.

\begin{tabular}{|c|c|c|c|c|c|c|}
\hline Autor/Ano & Título & $\begin{array}{l}\text { Perió- } \\
\text { dico }\end{array}$ & Objetivo & Método & Resultados & $\begin{array}{l}\text { Nivel } \\
\text { de Evi- } \\
\text { dência }\end{array}$ \\
\hline $\begin{array}{l}\text { Kent- } \\
\text { Wilkinson, } \\
2009 .\end{array}$ & $\begin{array}{l}\text { Forensic nursing } \\
\text { education in North } \\
\text { America: Social } \\
\text { factors influencing } \\
\text { educational devel- } \\
\text { opment }\end{array}$ & $\begin{array}{l}\text { Journal } \\
\text { of Fo- } \\
\text { rensic } \\
\text { Nursing }\end{array}$ & $\begin{array}{l}\text { Explorar o conhecimento } \\
\text { forense de enfermagem } \\
\text { como área especializada } \\
\text { de estudo e fatores que in- } \\
\text { fluenciam o desenvolvimen- } \\
\text { to educacional. }\end{array}$ & $\begin{array}{l}\text { Pesquisa Quali- } \\
\text { tativa }\end{array}$ & $\begin{array}{l}\text { Mudanças no contexto social impactam } \\
\text { na prática de enfermagem forense aos } \\
\text { profissionais. }\end{array}$ & 4 \\
\hline $\begin{array}{l}\text { Kent- } \\
\text { Wilkinson, } \\
2009 .\end{array}$ & $\begin{array}{l}\text { An exploratory } \\
\text { study of forensic } \\
\text { nursing education } \\
\text { in North America: } \\
\text { Constructed defi- } \\
\text { nitions of forensic } \\
\text { nursing }\end{array}$ & $\begin{array}{l}\text { Journal } \\
\text { of Fo- } \\
\text { rensic } \\
\text { Nursing }\end{array}$ & $\begin{array}{l}\text { Explorar o conhecimento } \\
\text { forense de enfermagem } \\
\text { como área especializada } \\
\text { de estudo, e fatores que } \\
\text { influenciam seu desenvolvi- } \\
\text { mento educacional. }\end{array}$ & $\begin{array}{l}\text { Pesquisa Quali- } \\
\text { tativa }\end{array}$ & $\begin{array}{l}\text { Houve diferentes conceitos sobre a en- } \\
\text { fermagem forense e sua relevância como } \\
\text { ciência. }\end{array}$ & 4 \\
\hline $\begin{array}{l}\text { Silva KB, Silva } \\
\text { R de C, } 2009 .\end{array}$ & $\begin{array}{l}\text { Enfermagem foren- } \\
\text { se: uma especialida- } \\
\text { de a conhecer }\end{array}$ & $\begin{array}{l}\text { Cogitare } \\
\text { Enferm. }\end{array}$ & $\begin{array}{l}\text { Relatar como ocorre a atua- } \\
\text { ção da enfermagem foren- } \\
\text { se nos Estados Unidos da } \\
\text { América }\end{array}$ & $\begin{array}{l}\text { Revisão de Lite- } \\
\text { ratura }\end{array}$ & $\begin{array}{l}\text { Contribuição para trazer a especialidade } \\
\text { de enfermagem forense ao Brasil. }\end{array}$ & 6 \\
\hline $\begin{array}{l}\text { Ferguson CT, } \\
\text { Speck PM, } \\
2010 \text {. }\end{array}$ & $\begin{array}{l}\text { The forensic nurse } \\
\text { and violence pre- } \\
\text { vention and re- } \\
\text { sponse in public } \\
\text { health }\end{array}$ & $\begin{array}{l}\text { Journal } \\
\text { of Fo- } \\
\text { rensic } \\
\text { Nursing }\end{array}$ & $\begin{array}{l}\text { Prevenir a violência através } \\
\text { da resposta do enfermeiro } \\
\text { forense na saúde pública. }\end{array}$ & $\begin{array}{l}\text { Revisão de Liter- } \\
\text { atura }\end{array}$ & $\begin{array}{l}\text { Mudanças no ensino da enfermagem fo- } \\
\text { rense na atuação frente ao combate à } \\
\text { violência. }\end{array}$ & 6 \\
\hline $\begin{array}{l}\text { Kent- } \\
\text { Wilkinson, } \\
2011 .\end{array}$ & $\begin{array}{l}\text { Forensic nursing } \\
\text { educational devel- } \\
\text { opment: An inte- } \\
\text { grated review of the } \\
\text { literature }\end{array}$ & $\begin{array}{l}\text { Journal } \\
\text { of Psy- } \\
\text { chiatric } \\
\text { and } \\
\text { Mental } \\
\text { Health } \\
\text { Nursing }\end{array}$ & $\begin{array}{l}\text { Analisar o desenvolvimento } \\
\text { histórico de cada uma das } \\
\text { subespecialidades de enfer- } \\
\text { magem forense. }\end{array}$ & $\begin{array}{l}\text { Revisão Inte- } \\
\text { grativa }\end{array}$ & $\begin{array}{l}\text { Identificou déficits de conhecimento so- } \\
\text { bre a enfermagem forense como espe- } \\
\text { cialidade. }\end{array}$ & 6 \\
\hline $\begin{array}{l}\text { Alsaif DM et } \\
\text { al., } 2014 .\end{array}$ & $\begin{array}{l}\text { Forensic experience } \\
\text { of Saudi nurses; An } \\
\text { emerging need for } \\
\text { forensic qualifica- } \\
\text { tions }\end{array}$ & $\begin{array}{l}\text { Journal } \\
\text { of Fo- } \\
\text { rensic } \\
\text { and } \\
\text { Legal } \\
\text { Medicine }\end{array}$ & $\begin{array}{l}\text { Determinar as qualifica- } \\
\text { ções forenses de enfermei- } \\
\text { ros que atuam em serviços } \\
\text { de emergência na área de } \\
\text { Dammam e seus conheci- } \\
\text { mentos sobre os principios } \\
\text { da enfermagem forense. }\end{array}$ & $\begin{array}{l}\text { Pesquisa Analiti- } \\
\text { ca Descritiva }\end{array}$ & $\begin{array}{l}\text { A escassez de conhecimento sobre a } \\
\text { enfermagem forense faz com que os en- } \\
\text { fermeiros busquem se capacitarem com } \\
\text { intuito de melhore a assistência de tra- } \\
\text { balho. }\end{array}$ & 4 \\
\hline $\begin{array}{l}\text { Choi KC, } \\
2014 .\end{array}$ & $\begin{array}{l}\text { Forensic Nursing } \\
\text { Philosophy: Examin- } \\
\text { ing Epistemological } \\
\text { Assumptions About } \\
\text { Truth and Knowl- } \\
\text { edge in the Inte- } \\
\text { grated } \\
\text { Practice Model }\end{array}$ & $\begin{array}{l}\text { Journal } \\
\text { of Fo- } \\
\text { rensic } \\
\text { Nursing }\end{array}$ & $\begin{array}{l}\text { Esclarecer as definições de } \\
\text { verdade e o conceito rela- } \\
\text { cionado ao conhecimento } \\
\text { na enfermagem forense. }\end{array}$ & Editorial & $\begin{array}{l}\text { Importância da teoria da enfermagem } \\
\text { forense para a disseminação do conheci- } \\
\text { mento aos enfermeiros }\end{array}$ & 6 \\
\hline
\end{tabular}




\begin{tabular}{|c|c|c|c|c|c|c|}
\hline Autor/Ano & Título & $\begin{array}{l}\text { Perió- } \\
\text { dico }\end{array}$ & Objetivo & Método & Resultados & $\begin{array}{l}\text { Nivel de } \\
\text { Evidên- } \\
\text { cia }\end{array}$ \\
\hline $\begin{array}{l}\text { Esteves RB, } \\
\text { Laiuk GC, } \\
\text { Cardoso } \\
\text { L. Kent- } \\
\text { Wilkinson A, } \\
2014 .\end{array}$ & $\begin{array}{l}\text { Toward the establis- } \\
\text { hment of a forensic } \\
\text { nursing specialty in } \\
\text { Brazil: An integrative } \\
\text { literature review }\end{array}$ & $\begin{array}{l}\text { Journal } \\
\text { of Fo- } \\
\text { rensic } \\
\text { Nursing }\end{array}$ & $\begin{array}{l}\text { Analisar e sintetizar sobre o desenvol- } \\
\text { vimento da especialidade enfermagem } \\
\text { forense em todo o mundo e extrair } \\
\text { lições para o estabelecimento impor- } \\
\text { tante de uma especialidade de enfer- } \\
\text { magem forense no Brasil. }\end{array}$ & $\begin{array}{l}\text { Revisão } \\
\text { Integra- } \\
\quad \text { tiva }\end{array}$ & $\begin{array}{l}\text { Contribuição da enfermagem foren- } \\
\text { se internacional para a enfermagem } \\
\text { brasileira. }\end{array}$ & 6 \\
\hline $\begin{array}{l}\text { Kalayci I, } \\
\text { Yazici SO, } \\
\text { Küpeli A, } \\
2014 .\end{array}$ & $\begin{array}{l}\text { Assesment of the } \\
\text { Knowledge Level of } \\
\text { Nursing Students } \\
\text { on Forensic Nursing }\end{array}$ & $\begin{array}{l}\text { Procedia } \\
\text { - Social } \\
\text { and Be- } \\
\text { havioral } \\
\text { Sciences }\end{array}$ & $\begin{array}{l}\text { Avaliar o nivel de conhecimento de es- } \\
\text { tudantes de enfermagem em Saúde da } \\
\text { Escola da Universidade Mehmet Akif } \\
\text { Ersoy sobre o conceito de enfermagem } \\
\text { forense. }\end{array}$ & $\begin{array}{l}\text { Pesquisa } \\
\text { Trans- } \\
\text { versal } \\
\text { Descri- } \\
\text { tiva }\end{array}$ & $\begin{array}{l}\text { Os estudantes não possuem conhe- } \\
\text { cimento sobre a disciplina de medici- } \\
\text { na forense e conhecimentos sobre a } \\
\text { enfermagem forense, possibilitando } \\
\text { a inserção de conteúdos curricula- } \\
\text { res. }\end{array}$ & 4 \\
\hline $\begin{array}{l}\text { Topçu ET, } \\
\text { Kazan EE, } \\
2018 .\end{array}$ & $\begin{array}{l}\text { The opinions of seni- } \\
\text { or nursing students } \\
\text { about forensic nur- } \\
\text { sing }\end{array}$ & $\begin{array}{l}\text { Egyptian } \\
\text { Jour- } \\
\text { nal of } \\
\text { Forensic } \\
\text { Sciences }\end{array}$ & $\begin{array}{l}\text { Determinar as opiniões de estudantes } \\
\text { de enfermagem seniores sobre enfer- } \\
\text { magem forense. }\end{array}$ & $\begin{array}{l}\text { Pesquisa } \\
\text { Descri- } \\
\text { tiva }\end{array}$ & $\begin{array}{l}\text { A enfermagem forense deveria ser } \\
\text { oferecida como uma disciplina nas } \\
\text { intuições de ensino, e ter seu reco- } \\
\text { nhecimento como especialidade e } \\
\text { capacitar os profissionais a respeito } \\
\text { dessa ciência. }\end{array}$ & 4 \\
\hline $\begin{array}{l}\text { Simmons B, } \\
2014 .\end{array}$ & $\begin{array}{l}\text { Graduate forensic } \\
\text { nursing education: } \\
\text { How to better edu- } \\
\text { cate nurses to care } \\
\text { for this patient pop- } \\
\text { ulation }\end{array}$ & $\begin{array}{l}\text { Nurse } \\
\text { Educator }\end{array}$ & $\begin{array}{l}\text { Fornecer informações sobre enferma- } \\
\text { gem forense e apresentar um modelo } \\
\text { de um programa de pós-graduação de } \\
\text { enfermagem forense que pode ser usa- } \\
\text { do como um guia de currículo. }\end{array}$ & $\begin{array}{l}\text { Revisão } \\
\text { da Lite- } \\
\text { ratura }\end{array}$ & $\begin{array}{l}\text { Contribuir para a formação de en- } \\
\text { fermeiros forenses por meio de } \\
\text { programas de ensino, a melhoria do } \\
\text { cenário da prática. }\end{array}$ & 6 \\
\hline $\begin{array}{l}\text { Valentine } \\
\text { JL, } 2014 .\end{array}$ & $\begin{array}{l}\text { Why we do what we } \\
\text { do: A theoretical } \\
\text { evaluation of the } \\
\text { integrated practice } \\
\text { model for forensic } \\
\text { nursing science }\end{array}$ & $\begin{array}{l}\text { Journal } \\
\text { of Fo- } \\
\text { rensic } \\
\text { Nursing }\end{array}$ & $\begin{array}{l}\text { Explorar a teoria, aplicando diretrizes de } \\
\text { avaliação específicas e avaliar sua apli- } \\
\text { cação ao estado atual da enfermagem } \\
\text { forense. }\end{array}$ & $\begin{array}{l}\text { Revisão } \\
\text { da Lite- } \\
\text { ratura }\end{array}$ & $\begin{array}{l}\text { Mostra a relevância do modelo da } \\
\text { teoria da enfermagem forense para } \\
\text { o cenário atual da enfermagem, } \\
\text { como um ponto estratégico na mu- } \\
\text { dança do cenário científico. }\end{array}$ & 6 \\
\hline $\begin{array}{l}\text { Dash SK, } \\
\text { Patel S, } \\
\text { Chavali K, } \\
2016 .\end{array}$ & $\begin{array}{l}\text { Forensic nursing - } \\
\text { Global scenario and } \\
\text { Indian perspective }\end{array}$ & $\begin{array}{l}\text { Journal } \\
\text { of Foren- } \\
\text { sic and } \\
\text { Legal } \\
\text { Medicine }\end{array}$ & $\begin{array}{l}\text { Analisar a perspectiva da enfermagem } \\
\text { forense no cenário indiano. }\end{array}$ & $\begin{array}{l}\text { Revisão } \\
\text { de Lite- } \\
\text { ratura }\end{array}$ & $\begin{array}{l}\text { Lacunas de conhecimento no campo } \\
\text { de atuação e de formação, incenti- } \\
\text { vando os órgãos públicos e futuros } \\
\text { profissionais da área, em se especia- } \\
\text { lizarem no campo forense. }\end{array}$ & 6 \\
\hline
\end{tabular}


A partir da análise qualitativa dos estudos, surgiram duas categorias que serão discutidas a seguir, que envolvem: a) o ensino de enfermagem forense no campo assistencial e da pesquisa e b) o cenário da enfermagem forense como especialidade na enfermagem.

\section{DISCUSSÃO}

\section{O ensino de enfermagem forense no campo assistencial e da pesquisa}

A atuação do enfermeiro forense deve ser humanizada e integrada aos pacientes ${ }^{12}$. No entanto, esse profissional enfrenta no campo de trabalho problemas sociais e comportamentais referentes às vítimas, suspeitos ou agressores da violência ${ }^{13}$. Ter a sua presença na prática clínica pode trazer transformações positivas nos serviços de saúde, garantindo, assim, uma qualidade na assistência ${ }^{14}$. Para que isso ocorra, é imprescindivel que se formem profissionais conceituados para atender às necessidades atuais e futuras daqueles que precisam de cuidados ${ }^{15}$

No que se refere à formação dos profissionais de enfermagem, estudos ressaltam que a enfermagem forense pode impactar positivamente na formação dos discentes e dos docentes, incentivando-os no campo da pesquisa, com o intuito de melhorar não só o atendimento a casos de violência como também contribuir com a sociedade ${ }^{12-13}$.

Em vista disso, o ensino e a pesquisa são caminhos para que os enfermeiros adquiram autonomia, responsabilidade, diante dos enfrentamentos relacionados ao atendimento às vítimas e aos agressores da violência nos seus diferentes cenários ${ }^{15}$. Quando esses estudantes e profissionais adquirem conhecimentos em enfermagem forense, melhoram suas competências e habilidades para o atendimento às pessoas em situações de violência e despertam o interesse pela área, o que propicia dar continuidade em seus estudos, como no mestrado e no doutorado ${ }^{14}$.

A teoria de Virginia Lynch impacta na evolução e no crescimento da ciência da enfermagem forense, relaciona conceitos teóricos voltados às questões socioculturais, legislativas, comportamentais e de proteções as vítimas. Esse fato manifesta os aspectos positivos na reformulação de projetos pedagógicos de cursos de enfermagem ${ }^{14}$. Corrobora com o estudo que evidencia resultados satisfatórios ${ }^{16}$ daqueles que recebem informação e capacitação, porém existem ainda lacunas de conhecimentos sobre essa especialidade aos futuros enfermeiros ${ }^{17}$.

Alguns artigos evidenciam a lacunas de conhecimento de estudantes de enfermagem quanto a conceitos de enfermagem forense, campo de atuação profissional, assuntos relacionados à medicina legal ${ }^{18-19}$.

Em visto disso, é necessário que as universidades invistam em programas de ensino, pesquisa e extensão que acrescentem ou valorizem os conteúdos curriculares, diante da necessidade de os enfermeiros buscarem programas de especialização na área de medicina legal bem como façam pesquisas para melhor reconhecimento dessa área nos campo de assistência e do ensino de enfermagem ${ }^{17,18,19}$.

\section{O cenário da enfermagem forense como especialidade na enfermagem}

Como especialidade, a enfermagem forense possibilita aprofundamento nas temáticas de violência quanto à avaliação e à assistência ${ }^{17}$, às políticas públicas de prevenção e de promoção de saúde ${ }^{16}$, aos aspectos do cuidado médico-legais da violência ${ }^{19}$ e à educação preventiva ${ }^{20}$.

Os resultados da enfermagem forense como espacialização se mostram positivos em vários países segundo as peculiaridades destes. Na Índia, a atuação está voltada para o atendimento de casos de violência sexual, destacando-se o papel do Enfermeiro Examinador de Agressão Sexual (SANE) ${ }^{21}$ esta permanece pouco explorada e reconhecida, ressaltando-se sua importância na assistência, no ensino e na pesquisa como forma de contribuir com a sociedade e com a justiça. $\mathrm{Na}$ Arábia Saudita, os profissionais que trabalham na emergência conhecem a especialidade e a prática forense, e a relevância desse profissional nesse setor. No entanto, 90,0\% deles não tiveram treinamentos, preparo ou educação continuada para lidarem com casos forenses em seu ambiente de trabalho ${ }^{17}$.

Nas regiões do Canadá, EUA, Bermuda, Puerto Rico e Austrália, destacam-se os Enfermeiros Forenses Examinadores de Agressão Sexual (SANE) no atendimento a crianças, adolescentes e adultos ${ }^{14,20,22,23}$.

Na América do Sul, o Brasil é um dos países em que o avanço dessa especialidade está sendo consolidado, sendo um campo ainda pouco explorado nos cursos de enfermagem, o que levanta lacunas pela falta de conhecimentos nos cursos e a necessidade de falar sobre o assunto nas universidades ${ }^{24}$. Acrescenta-se que essa é uma oportunidade para a enfermagem brasileira avançar e implantar a especialidade no país (graduação e pós-graduação), para atender às necessidades da população, seja no campo social, cultural, econômico e político, bem como melhorar a assistência de enfermagem a esse público, para que os profissionais consigam desenvolver habilidades, competências e autonomia no cuidado 22 .

Quanto à oferta de cursos de enfermagem forense, existem aspectos positivos que estão relacionados à oportunidade e ao interesse de quererem se especializar, no entanto há aspectos negativos voltados à qualidade da oferta desses cursos e à falta de oportunidade ao mercado de trabalho para esses especialistas, impactando não somente 
na formação desses profissionais, como em sua assistência frente as situações de violência e de criminalidade ${ }^{20}$

\section{LIMITAÇÕES DO ESTUDO}

Como limitações do estudo, evidenciam-se poucos trabalhos sobre o tema, o que motiva a realização de pesquisas que possam contribuir para a enfermagem forense na graduação, com estudos que mostrem as lacunas de conhecimento e suas reflexões na assistência para que se possam desenvolver habilidades e competências ainda no processo de formação do enfermeiro. No contexto social, essa realidade também merece atenção uma vez que essas lacunas inviabilizam uma prática assistencial resolutiva.

\section{Contribuições do Estudo para a Prática}

O estudo contribui para despertar nos cursos de enfermagem a possibilidade de acrescentar uma disciplina de Enfermagem forense nos respectivos cursos, mesmo sendo considerada uma especialização da profissão, ela pode impactar positivamente na formação dos discentes, no incentivo a pesquisas e no cuidado integral a população.

\section{CONCLUSÃO}

Por meio da revisão de literatura, foi possível explorar o cenário da enfermagem forense na formação do enfermeiro na assistência e na pesquisa. Evidenciou-se que a enfermagem forense é relativamente nova enquanto especialidade, mas pode contribuir na formação e na emancipação da assistência e na pesquisa em enfermagem.

A enfermagem forense tem-se apresentado como facilitadora da assistência ética, humanística e integradora tanto para as vítimas de violência quanto para os perpetradores. Os enfermeiros examinadores de agressão sexual (SANE) se destacaram como campo de atuação da enfermagem em diferentes países. Reconhece-se que, no Brasil, estudos devem ser desenvolvidos a fim de propiciar a identificação de novos campos de inserção dos profissionais de enfermagem.

Contribuição dos Autores: Jhuliano Silva Ramos de Souza: concepção e/ou desenho; análise e interpretação dos dados; redação do artigo; revisão crítica e revisão final; Andreia Cristina Barbosa Costa: análise e interpretação dos dados; redação do artigo; revisão crítica; Sueli de Carvalho Vilela: concepção e/ou desenho; análise e interpretação dos dados; redação do artigo; revisão crítica e revisão final.

Financiamento: $O$ presente trabalho foi realizado com o apoio da Coordenação de Aperfeiçoamento de Pessoal de Nivel Superior (CAPES) - Código de Financiamento 001.

\section{REFERÊNCIAS}

1. Nunes ECDA, Silva LWS, Pires EPOR. Nursing Professional education: implications of education for transpersonal care. Rev. Latino-Am. Enfermagem. 2011; 19(2):252-6. doi: https://dx.doi.org/10.1590/S010411692011000200005

2. International Association of Forensic Nurses. History of the Association. Silver Spring, Maryland: IAFN, 2017. Disponivel em: https://www.forensicnurses.org/page/AboutUS? Acesso em: 22/01/2020.

3. Conselho Federal de Enfermagem. Resolução nㅇ 389, de outubro de 2011. Procedimentos de título de pós-graduação lato e stricto sensu concedido a enfermeiros e lista as especialidades. 202ㅇ - Seção 1- p-146. Brasilia: Cofen, 2011. Disponivel em: http://www.cofen.gov.br/wp-content/uploads/2012/03/ resolucao_389_2011.pdf Acesso em: 21/01/2020.

4. Lynch VA. Forensic nursing science: global strategies in health and justice. Egyptian Journal of Forensic Sciences. 2011; 1(2):69-76. doi: https://dx.doi.org/10.1016/j.ejfs.2011.04.001

5. Kent-Wilkinson A. An exploratory study of forensic nursing education in north america: constructed definitions of forensic nursing. J Forensic Nurs. 2009:5(4):201-11. doi: https://dx. doi.org/ 10.1111/j.19393938.2009.01055.x

6. Cerqueira D, Bueno S, Lima RSD, Neme C, Ferreira H, Alves PP et al. Atlas da Violência 2019 - Ipea e FBSP. Brasília, DF: Instituto de Pesquisa Econômica Aplicada; 2019. 116 p. Disponível em: http://www.ipea.gov. br/portal/images/stories/PDFs/relatorio_institucional/190605_atlas_da_violencia_2019.pdf Acesso em: $30 / 08 / 2019$. 
7. Simmons B, Grandfield K. Focus on forensic nursing education. J Emerg Nurs. 2013;39(6):633-4. doi: https:// dx.doi.org/ 10.1016/j.jen.2013.06.010

8. Mendes KDS, Silveira RCCP, Galvão CM. Revisão integrativa: método de pesquisa para a incorporação de evidências na saúde e na enfermagem. Texto contexto - enferm. 2008; 17(4): 758-764. doi: https://dx.doi. org/10.1590/S0104-07072008000400018

9. Stetler CB, Morsi D, Rucki S, Broughton S, Corrigan B, Fitzgerald J, et al. Utilization-focused integrative reviews in a nursing service. Appl Nurs Res. 1998;11(4):195-206. doi: https://dx.doi.org/ 10.1016/s08971897(98)80329-7

10. Bardin L. Análise de conteúdo. Trad. de Luís Antero Reto e Augusto Pinheiro.70. ed. Lisboa; 2010.

11. Moher D, Liberati A, Tetzlaff J, Altman DG. The PRISMA Group 2009.Preferred reporting items for systematic reviews and meta-analyses: the PRISMA Statement. PLoS Med. 2009; 6(6):el000097.doi: https:// dx.doi.org/10.1371/journal.pmed.1000097

12. Choi KR. Forensic nursing philosophy: examining epistemological assumptions about truth and knowledge in the integrated practice model. J Forensic Nurs. 2014;10(4):243-4. doi: https://dx.doi.org/10.1097/ JFN.0000000000000050

13. Valentine JL. Why we do what we do: A theoretical evaluation of the integrated practice model for forensic nursing science. J Forensic Nurs. 2014;10(3):113-9. doi: https://dx.doi.org/ 10.1097/JFN.0000000000000041

14. Simmons B. Graduate forensic nursing education: how to better educate nurses to care for this patient population. Nurse Educ. 2014;39(4):184-7. doi: https://dx.doi.org/ 10.1097/ NNE.0000000000000047

15. Sekula LK, Colbert AM, Zoucha R, Amar AF, Williams J. Strengthening the science of forensic nursing through education and research. J Forensic Nurs. 2012;8(1):1-2. doi: https://dx.doi.org/10.1111/j.19393938.2012.01136.x

16. Ferguson LCT, Speck PM. The forensic nurse and violence prevention and response in public health. $\mathrm{J}$ Forensic Nurs. 2010;6(3):151-6. doi: https://dx.doi.org/10.1111/j.1939-3938.2010.01080.x

17. Alsaif DM, Alfaraidy M, Alsowayigh K, Alhusain A, Almadani OM. Forensic experience of saudis nurses: an emerging need for forensic qualifications. J Forensic Leg Med. 2014; 27:13-6. doi: http://dx.doi.org/10.1016/j. jflm.2014.07.004

18. Topçu ET, Kazan EE. The opinions of senior nursing students about forensic nursing. Egypt J Forensic Sci. 2018;8(1). doi: https://dx.doi.org/ 10.1186/s41935-018-0045-y

19. Kalayci I, Yazici SO, Kupeli A. Assesment of the knowledge level of nursing students on forensic nursing. Procedia - Soc Behav Sci.2014; 131:130-4. doi: https://dx.doi.org/ 10.1016/j.sbspro.2014.04.091

20. Kent-Wilkinson AE. Forensic nursing education in north america: social factors influencing educational development. J Forensic Nurs. 2009;5(2):76-88. doi: https://dx.doi.org/10.1111/j.1939-3938.2009.01038.x

21. Dash SK, Patel S, Chavali K. Forensic nursing: global scenario and indian perspective. J Forensic Leg Med. 2016; 42:88-91. doi: http://dx.doi.org/10.1016/j.jflm.2016.05.020

22. Esteves RB, Lasiuk GC, Cardoso L, Kent WA. Toward the establishment of a forensic nursing specialty in Brazil: An integrative literature review. J Forensic Nurs. 2014;10(4):189-98. doi: https://dx.doi.org./ 10.1097/ JFN.0000000000000048

23. Kent-Wilkinson A. Forensic nursing educational development: an integrated review of the literature. $J$ Psychiatr Ment Health Nurs. 2011;18(3):236-46. doi: https://dx.doi.org/ 10.1111/j.1365-2850.2010.01667.x

24. Silva KB, Silva RDC. Enfermagem forense: uma especialidade a conhecer. Cogitare Enferm. 2009;14(3):5648. doi: ttp://dx.doi.org/10.5380/ce.v14i3.16191 